\title{
First passage time density of an Ornstein-Uhlenbeck process with broken drift
}

\author{
Stefan Ankirchner* Christophette Blanchet-Scalliet ${ }^{\dagger}$ \\ Diana Dorobantu $u^{\ddagger}$ Laura Gay ${ }^{\S}$
}

March 4, 2021

\begin{abstract}
We consider an Ornstein-Uhlenbeck process with different drift rates below and above zero. We derive an analytic expression for the density of the first time, where the process hits a given level. The passage time density is linked to the joint law of the process and its running supremum, and we also provide an analytic formula of the joint density / distribution function. Results from a numerical experiment reveal that our formulas allow to numerically evaluate the joint law and the density of the first passage time faster than a simulation based method.
\end{abstract}

Keywords. Ornstein-Uhlenbeck process, piecewise-linear drift, first passage time density, Laplace transform, joint law of the running supremum and endpoint, Fokker-Planck equation.

\section{Introduction}

In this article we consider an Ornstein-Uhlenbeck (OU) process with a drift coefficient that is linear above and below zero, but with different rates. The drift coefficient is thus a piecewise linear function with a break point at zero. The aim is to determine the law of the first time the process hits a given level, and the joint law of the process and its running supremum. For the classical Ornstein-Uhlenbeck process with a globally linear drift these laws are already known: the distribution of first hitting times is computed in [2] and [13], and the joint law of the process and its running supremum in [3].

Ornstein-Uhlenbeck processes with piecewise linear drift coefficients have hardly been examined yet. If the slope is always positive, then the OU process has a stationary

\footnotetext{
*University of Jena, Institute for Mathematics, Germany, s.ankirchner@uni-jena.de

†University of Lyon, CNRS UMR 5208, Ecole Centrale de Lyon, Institut Camille Jordan, France, christophette.blanchet@ec-lyon.fr

$\ddagger$ University of Lyon, University Lyon 1, ISFA, LSAF (EA 2429) France, diana.dorobantu@univ-lyon1.fr

$\S$ University of Lyon, University Lyon 3, IAE, France, laura.gay@univ-lyon3.fr
} 
density, explicitly computed, e.g. in [4]. Apart from that, little is known, in particular about first hitting times. We show that if there is only one kink in the drift, then one can derive an analytic formula for the density of the first time the process attains a fixed level. Moreover, one can explicitly describe the joint law of the process and its running supremum.

First hitting times are intensively studied within applied probability theory for various reasons. They can be used, for example, for a probabilistic representation of parabolic PDEs with finite boundary conditions. In economic applications first hitting times are frequently used for modeling the arrival time of events, e.g. death times or bankruptcy times. In queuing theory diffusion hitting times can be used for approximating the first time a queue attains a certain length.

The recent years have seen an increased interest in diffusion processes with piecewise linear drift and piecewise constant diffusion coefficients. Such processes naturally arise, for example, when considering diffusion approximations of some queues (see [4]). Moreover, in many control problems the optimally controlled state is a diffusion with a piecewise linear drift or piecewise constant diffusion coefficient, respectively. In particular, the OU process with a single kink in its drift can be interpreted as an optimally controlled state process within a model where an agent aims at maximizing the process's occupation time above zero (see Section 4 in [7]). A Brownian motion with piecewise constant diffusion coefficients appears in optimal diffusion control problems with piecewise convex and concave payoff functions (see [14]).

Several authors have considered some optimization problems within models using diffusions with piecewise linear drift and piecewise constant diffusion coefficients. For example in [16] and [15], the authors solve optimal stopping problems for a Brownian motion with positive piecewise constant volatility $([16])$ or a positive piecewise constant drift ([15]) changing at zero. Another optimal stopping problem related to the pricing of an American option in an extension of the Black-Merton-Scholes model with piecewise constant dividend and volatility rates appears in [6].

A statistical perspective is taken in [12], analyzing a maximum likelihood estimator for diffusions with piecewise constant drift and diffusion coefficients. The benefit of diffusions with piecewise constant parameters in financial applications is demonstrated in [8]. The authors consider a version of the Heston model with piecewise constant parameters and discuss its calibration to the foreign exchange options market.

We obtain our results by studying the Fokker-Planck equation associated to the joint law of the process and its running supremum. We explicitly solve the Fokker-Planck equation with the spectral method, and thus obtain an infinite series representation of the joint density/ distribution function of the process and its supremum at a given time $t \in[0, \infty)$. A rather short calculation then allows to derive, from the joint law, a density formula for first passage times. 
We remark that an alternative way for determining hitting time densities is to compute first their Laplace transform and then to use a Laplace inversion theorem. Indeed, the Laplace transform of the first passage time of the OU with a single kink in its drift can be calculated explicitly (see Section 4 in [7]). From this one can, formally, derive an analytic formula for the density of the first passage time. The Laplace transform, however, has infinitely many poles and thus the inversion requires to determine the residues along these poles. It seems to us that is is difficult to check whether the conditions of the residue theorem are satisfied, and therefore we do not follow this path.

The paper is organized as follows. In Section 2 we present the expressions of the density for the first passage time of an Ornstein-Uhlenbeck process with broken drift. We also describe the joint density / distribution function of the process and its running supremum. Moreover, we present some results from numerical experiments. Section 3 is devoted to the proof of our main results. For the reader's convenience, in Section 4 we provide some properties and auxiliary results of Hermite functions and parabolic cylinder functions.

\section{Results}

\subsection{Ornstein-Uhlenbeck process with broken drift}

Let $\left(B_{t}\right)_{t \geq 0}$ be a standard Brownian motion defined on a probability space $(\Omega, \mathcal{F}, \mathbb{P})$. Moreover let $k_{-}, k_{+} \in \mathbb{R}_{+}^{*}$ and let $k: \mathbb{R} \rightarrow \mathbb{R}$ be the function defined by

$$
k(x)= \begin{cases}k_{-}, & \text {if } x \leq 0 \\ k_{+}, & \text {if } x>0\end{cases}
$$

Let $x_{0} \in \mathbb{R}$ and denote by $X=\left(X_{t}\right)_{t \geq 0}$ the strong solution of the stochastic differential equation $(\mathrm{SDE})$

$$
\mathrm{d} X_{t}=-k\left(X_{t}\right) X_{t} \mathrm{~d} t+\mathrm{d} B_{t}, \quad X_{0}=x_{0} .
$$

Note that the drift coefficient of $X$ is a piecewise linear function with a kink in zero. In accordance with [15] we refer to $X$ as an Ornstein-Uhlenbeck process with a broken drift.

Let $m \in \mathbb{R}$ be fixed. We introduce the first passage time of $\left(X_{t}\right)_{t \geq 0}$ of the level $m$ :

$$
\tau=\inf \left\{t>0, X_{t}=m\right\}
$$

Note that if $x_{0}<m \leq 0$ or $x_{0}>m \geq 0$, then $\tau$ is the first passage time of a "classical" OU process with drift function $x \mapsto k_{-} x$ and $x \mapsto k_{+} x$, respectively. The only cases requiring new computations are $\left(x_{0} \leq m, m>0\right)$ and $\left(x_{0} \geq m, m<0\right)$. Symmetry allows to reduce the second case to the first one. Therefore, in the following we consider only the case $\left(x_{0} \leq m, m>0\right)$. 


\subsection{Main theorems}

Our first aim is to prove that $\tau$ has a density $p_{\tau}$ and to derive an analytic formula for it. We introduce the following notations: for any $\mu \in \mathbb{R}$ let $D_{\mu}$ be the parabolic cylinder function of the parameter $\mu$ (see Appendix 4.1 for the definition) and

$$
\alpha_{\mu}=\frac{1}{2}\left[\frac{D_{\frac{\mu}{k_{-}}}(0)}{D_{\frac{\mu}{k_{+}}}(0)}+\sqrt{\frac{k_{+}}{k_{-}}} \frac{D_{\frac{\mu}{k_{-}}-1}(0)}{D_{\frac{\mu}{k_{+}}-1}(0)}\right], \beta_{\mu}=\frac{1}{2}\left[\frac{D_{\frac{\mu}{k_{-}}}(0)}{D_{\frac{\mu}{k_{+}}}(0)}-\sqrt{\frac{k_{+}}{k_{-}}} \frac{D_{\frac{\mu}{k_{-}}-1}(0)}{D_{\frac{\mu}{k_{+}}-1}(0)}\right] .
$$

Theorem 1. We assume that $\left.\left.x_{0} \in\right]-\infty, m\right], m \in \mathbb{R}_{+}^{*}$. Then

1. The probability $\mathbb{P}(\tau \in \mathrm{d} t)$ admits a density $p_{\tau}$,

2. The density $p_{\tau}$ satisfies, for all $t \in \mathbb{R}_{+}^{*}$,

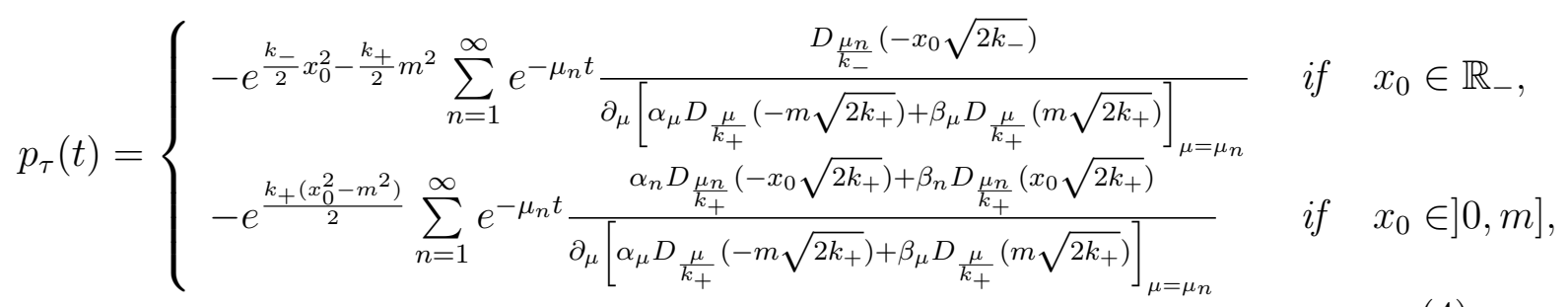

where $\alpha_{n}=\alpha_{\mu_{n}}, \beta_{n}=\beta_{\mu_{n}}$, and $\left(\mu_{n}\right)_{n \geq 1}$ is the ordered sequence of positive zeros of $\mu \mapsto \alpha_{\mu} D_{\frac{\mu}{k_{+}}}\left(-m \sqrt{2 k_{+}}\right)+\beta_{\mu} D_{\frac{\mu}{k_{+}}}\left(m \sqrt{2 k_{+}}\right)$.

We prove Theorem 1 by using an explicit expression for the joint density/distribution of the endpoint and the running supremum, more precisely for

$$
\mathbb{P}\left(X_{t} \in d x, \tau>t\right)=\mathbb{P}\left(X_{t} \in d x, \sup _{u \leq t} X_{u}<m\right) .
$$

The analytic formula for the joint density/distribution is given in the following theorem.

Theorem 2. We assume that $\left.\left.x_{0} \in\right]-\infty, m\right], m \in \mathbb{R}_{+}^{*}$. Then

1. For all $t \in \mathbb{R}_{+}^{*}$ the probability $\mathbb{P}\left(X_{t} \in \mathrm{d} x, \sup _{0 \leq u \leq t} X_{u} \leq m\right)$ admits a density $p$ with support in $]-\infty, m]$ :

$$
\mathbb{P}\left(X_{t} \in \mathrm{d} x, \sup _{0 \leq u \leq t} X_{u} \leq m\right)=p(m, x, t) \mathrm{d} x .
$$

2. The density $p$ solves the following Fokker-Planck equation:

$$
\left\{\begin{array}{clrl}
\partial_{t} p(m, x, t) & =k_{-} \partial_{x}(x p(m, x, t))+\frac{1}{2} \partial_{x^{2}}^{2} p(m, x, t), & (x, t) \in]-\infty, 0\left[\times \mathbb{R}_{+}^{*},\right. \\
\partial_{t} p(m, x, t) & =k_{+} \partial_{x}(x p(m, x, t))+\frac{1}{2} \partial_{x^{2}}^{2} p(m, x, t), & (x, t) \in] 0, m] \times \mathbb{R}_{+}^{*}, \\
p\left(m, 0^{-}, t\right) & =p\left(m, 0^{+}, t\right), & t \in \mathbb{R}_{+}^{*}, \\
\partial_{x} p\left(m, 0^{-}, t\right) & =\partial_{x} p\left(m, 0^{+}, t\right), & t \in \mathbb{R}_{+}^{*}, \\
p(m, m, t) & =0 & t \in \mathbb{R}_{+}^{*}, \\
\lim _{t \downarrow 0} p(m, x, t) d x & =\delta_{x_{0}}(d x) . &
\end{array}\right.
$$


3. With the notations of Theorem 1 and for $(m, x) \in\left\{(z, y) \in \mathbb{R}_{+}^{*} \times \mathbb{R} \mid z \geq y\right\}, t \in \mathbb{R}_{+}^{*}$, the density $p$ can be expressed as:

- If $x_{0} \in \mathbb{R}_{-}$, then $p(m, x, t)=$

$$
\begin{cases}e^{\frac{k_{-}}{2}\left(x_{0}^{2}-x^{2}\right)} \sum_{n=1}^{\infty} \frac{e^{-\mu_{n} t}}{N\left(\mu_{n}\right)} D_{\frac{\mu_{n}}{k_{-}}}\left(-x \sqrt{2 k_{-}}\right) D_{\frac{\mu_{n}}{k_{-}}}\left(-x_{0} \sqrt{2 k_{-}}\right) & , x \leq 0 \\ e^{\frac{k_{-}}{2} x_{0}^{2}-\frac{k_{+}}{2} x^{2}} \sum_{n=1}^{\infty} \frac{e^{-\mu_{n} t}}{N\left(\mu_{n}\right)} D_{\frac{\mu_{n}}{k_{-}}}\left(-x_{0} \sqrt{2 k_{-}}\right)\left[\alpha_{n} D_{\frac{\mu_{n}}{k_{+}}}\left(-x \sqrt{2 k_{+}}\right)+\beta_{n} D_{\frac{\mu_{n}}{k_{+}}}\left(x \sqrt{2 k_{+}}\right)\right] & , 0<x \leq m\end{cases}
$$

- If $\left.\left.x_{0} \in\right] 0, m\right]$, then $p(m, x, t)=$

$$
\left\{\begin{array}{cl}
e^{\frac{k_{+}}{2} x_{0}^{2}-\frac{k_{-}}{2} x^{2}} \sum_{n=1}^{\infty} \frac{e^{-\mu_{n} t}}{N\left(\mu_{n}\right)} D_{\frac{\mu_{n}}{k_{-}}}\left(-x \sqrt{2 k_{-}}\right)\left[\alpha_{n} D \frac{\mu_{n}}{k_{+}}\left(-x_{0} \sqrt{2 k_{+}}\right)+\beta_{n} D_{\frac{\mu_{n}}{k_{+}}}\left(x_{0} \sqrt{2 k_{+}}\right)\right] & , x \leq 0 \\
e^{\frac{k_{+}}{2}\left(x_{0}^{2}-x^{2}\right)} \sum_{n=1}^{\infty} \frac{e^{-\mu_{n} t}}{N\left(\mu_{n}\right)}\left[\alpha_{n} D \frac{\mu_{n}}{k_{+}}\left(-x \sqrt{2 k_{+}}\right)+\beta_{n} D \frac{\mu_{n}}{k_{+}}\left(x \sqrt{2 k_{+}}\right)\right] \times & , 0<x \leq m \\
{\left[\alpha_{n} D_{\frac{\mu_{n}}{k_{+}}}\left(-x_{0} \sqrt{2 k_{+}}\right)+\beta_{n} D_{\frac{\mu_{n}}{k_{+}}}\left(x_{0} \sqrt{2 k_{+}}\right)\right]} & , 0 m
\end{array}\right.
$$

where

$$
\begin{aligned}
N\left(\mu_{n}\right)= & \frac{1}{2} \partial_{x}\left[\alpha_{n} D \frac{\mu_{n}}{k_{+}}\left(-x \sqrt{2 k_{+}}\right)+\beta_{n} D_{\frac{\mu_{n}}{k_{+}}}\left(x \sqrt{2 k_{+}}\right)\right]_{x=m} \\
& \times \partial_{\mu}\left[\alpha_{\mu} D_{\frac{\mu}{k_{+}}}\left(-m \sqrt{2 k_{+}}\right)+\beta_{\mu} D_{\frac{\mu}{k_{+}}}\left(m \sqrt{2 k_{+}}\right)\right]_{\mu=\mu_{n}} .
\end{aligned}
$$

From Theorem 1 and 2 we can derive the law of the running supremum alone.

Corollary 3. Let $\left.\left.x_{0} \in\right]-\infty, m\right], m \in \mathbb{R}_{+}^{*}$. Then

$$
\begin{aligned}
& \mathbb{P}\left(\sup _{0 \leq u \leq t} X_{u} \leq m\right) \\
& =\left\{\begin{array}{lll}
-e^{\frac{k_{-}}{2} x_{0}^{2}-\frac{k_{+}}{2} m^{2}} \sum_{n=1}^{\infty} \frac{e^{-\mu_{n} t}}{\mu_{n}} \frac{D \frac{\mu_{n}}{k_{-}}\left(-x_{0} \sqrt{2 k_{-}}\right)}{\left.\partial_{\mu}\left[\alpha_{\mu} D \frac{\mu}{k_{+}}\left(-m \sqrt{2 k_{+}}\right)+\beta_{\mu} D \frac{\mu}{k_{+}}\left(m \sqrt{2 k_{+}}\right)\right]\right]_{\mu=\mu_{n}}} & \text { if } & x_{0} \in \mathbb{R}_{-}, \\
-e^{\frac{k_{+}\left(x_{0}^{2}-m^{2}\right)}{2}} \sum_{n=1}^{\infty} \frac{e^{-\mu_{n} t}}{\mu_{n}} \frac{\alpha_{n} D \frac{\mu_{n}}{k_{+}}\left(-x_{0} \sqrt{2 k_{+}}\right)+\beta_{n} D \frac{\mu_{n}}{k_{+}}\left(x_{0} \sqrt{2 k_{+}}\right)}{\partial_{\mu}\left[\alpha_{\mu} D \frac{\mu}{k_{+}}\left(-m \sqrt{2 k_{+}}\right)+\beta_{\mu} D \frac{\mu}{k_{+}}\left(m \sqrt{2 k_{+}}\right)\right]_{\mu=\mu_{n}}} & \text { if } & \left.\left.x_{0} \in\right] 0, m\right] .
\end{array}\right.
\end{aligned}
$$

\section{$2.3 \quad$ Numerical results}

Even though Formulas (4), (6) (or (7)) are infinite sums of special functions, it is easy to evaluate them numerically. 
In a numerical experiment we have evaluated the functions $t \mapsto p_{\tau}(t)$ and $x \mapsto$ $p(m, x, t)$ first with expression (4) and (6) (or (7)) and, second, with a Monte Carlo method using $1 \times 10^{6}$ simulations and $\Delta_{t}=10^{-6}$ in the Euler Scheme. Figures 1 and 2 plot, respectively, $p_{\tau}(t)$ and $p(m, x, t)$ for some parameters and initial laws with $T=1$.

The experiments reveal that the computation with our formula is faster than with a Monte Carlo method. Indeed, for the density depicted in Figure 1, the evaluation with our formula only lasts 4.09 seconds time, whereas the Monte-Carlo method requires more than 16 hours, using a processor $3.6 \mathrm{GHz}$ with 4 cores.

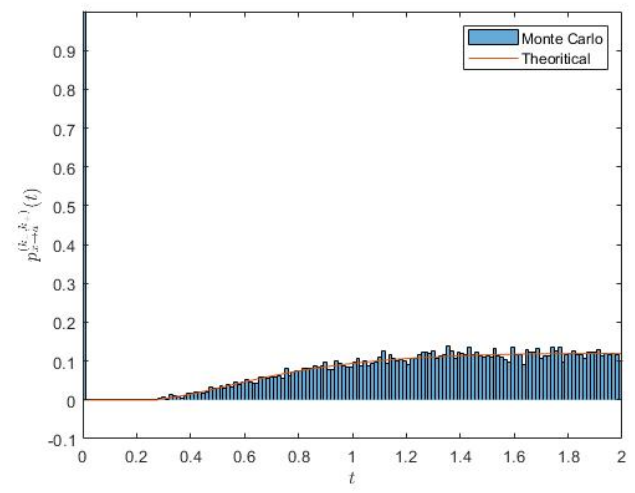

Figure 1: Comparison of expression (4) and a Monte Carlo method when $m=1$, $X_{0}=-1$ and $k_{+}=2, k_{-}=1$.

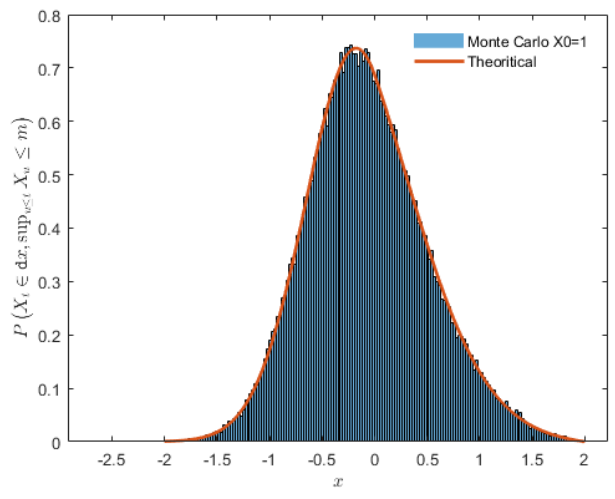

Figure 2: Comparison of expression (6) and a Monte Carlo method when $m=2$, $X_{0}=-1$ and $k_{+}=0.5, k_{-}=2$.

\section{Proofs}

This section is devoted to the proofs of Theorem 2, Theorem 1 and Corollary 3.

\subsection{Proof of Theorem 2}

1. The existence is given by Lemma 2.1. of [5] .

2. Directly from Proposition 5.4.3.1. of [9] .

3. We use a spectral method to solve the Fokker-Planck equation.

Let us note $D\left(\mathbf{L}_{F P}\right)$ the set

$$
\left.\left.\left.\left\{f \in L^{2}(]-\infty, m\right], e^{k(.) .^{2} / 2}\right) \mid f^{\prime} \in L^{2}(]-\infty, m\right], e^{k(.) .^{2} / 2}\right), f(m)=0, f\left(0^{+}\right)=f\left(0^{-}\right), f^{\prime}\left(0^{+}\right)=f^{\prime}\left(0^{-}\right),
$$


$\left.x \mapsto-k_{-}(x f(x))^{\prime}-\frac{1}{2} f^{\prime \prime}(x) \in L^{2}(]-\infty, 0\left[, e^{k_{-} \cdot{ }^{2} / 2}\right), x \mapsto-k_{+}(x f(x))^{\prime}-\frac{1}{2} f^{\prime \prime}(x) \in L^{2}(] 0, m\left[, e^{k_{+} \cdot{ }^{2} / 2}\right)\right\}$

The Fokker-Plank operator $\mathbf{L}_{F P}$ is defined by

$$
\begin{aligned}
\mathbf{L}_{F P}: D\left(\mathbf{L}_{F P}\right) & \left.\left.\longrightarrow L^{2}(]-\infty, m\right], e^{k(.) .^{2} / 2}\right) \\
f & \longrightarrow\left[\mathbf{L}_{F P} f: x \mapsto-(k(x) x f(x))^{\prime}-\frac{1}{2} f^{\prime \prime}(x)\right]
\end{aligned}
$$

Our goal is to find a basis $\left(e_{n}\right)_{n \geq 1}$ of eigenvectors of $\mathbf{L}_{F P}$ in order to write

$$
p(m, x, t)=\sum_{n=1}^{\infty} c_{n}(t) e_{n}(x)
$$

\section{Hilbertian basis of $\left.\left.L^{2}(]-\infty, m\right], e^{k(.) .^{2} / 2}\right)$}

Let remark that $\mathbf{L}_{F P}$ is Hermitian. We prove that any function of $\left.\left.L^{2}(]-\infty, m\right], e^{k(.) .^{2} / 2}\right)$ can be decomposed on an Hilbertian basis of eigenvectors of $\mathbf{L}_{F P}$. Since, it is not easy to prove it directly, we introduce a new operator $\mathbf{T}$ defined as

$$
\begin{aligned}
\left.\left.\mathbf{T}: L^{2}(]-\infty, m\right], e^{k(.)^{2} / 2}\right) & \left.\left.\longrightarrow L^{2}(]-\infty, m\right], e^{k(.) .^{2} / 2}\right) \\
g & \longrightarrow f_{g}
\end{aligned}
$$

where $f_{g}$ is solution of

$$
(*)=\left\{\begin{aligned}
-\frac{1}{2} f_{g}^{\prime \prime}-k_{-}\left(x f_{g}\right)^{\prime} & =g \text { on }]-\infty, 0[ \\
-\frac{1}{2} f_{g}^{\prime \prime}-k_{+}\left(x f_{g}\right)^{\prime} & =g \text { on }] 0, m[ \\
f_{g}(m) & =0 \\
f_{g}\left(0^{-}\right) & =f_{g}\left(0^{+}\right) \\
f_{g}^{\prime}\left(0^{-}\right) & =f_{g}^{\prime}\left(0^{+}\right) \\
f_{g}, f_{g}^{\prime} & \left.\left.\in L^{2}(]-\infty, m\right], e^{k(.))^{2} / 2}\right)
\end{aligned}\right.
$$

The eigenvectors of $\mathbf{T}$ are the same as those of $\mathbf{L}_{F P}$ with inverse eigenvalues. Since $\mathbf{T}$ and $\mathbf{L}_{F P}$ are Hermitian, we can use classical results on Hermitian operators decomposition. To prove that $\mathbf{T}$ is well defined we write it as an integral operator.

Lemma 1. The solution of $(*)$ is $f_{g}(x)=2 \int_{-\infty}^{m} K(x, z) g(z) d z$, where

$$
K(x, z)=\mathbf{1}_{x<0} \int_{x \vee z}^{m} e^{k_{-}\left(y^{2}-x^{2}\right)} d y+\mathbf{1}_{0 \leq x \leq m} \int_{x \vee z}^{m} e^{k_{+}\left(y^{2}-x^{2}\right)} d y .
$$


Proof. We remark that $f_{g}(m)=0$. We easily check that $f_{g}$ satisfies $(*)$ :

$$
\begin{aligned}
f_{g}^{\prime}(x) & =2 \int_{-\infty}^{m} \frac{\partial}{\partial x} K(x, z) g(z) d z \text { with } \\
\partial_{x} K(x, z) & =-2 k_{-} x K(x, z) \mathbf{1}_{x<0}-2 k_{+} x K(x, z) \mathbf{1}_{0 \leq x \leq m} \underbrace{-\mathbf{1}_{x>z} \mathbf{1}_{x<0}-\mathbf{1}_{x>z} \mathbf{1}_{0 \leq x \leq m}}_{-\mathbf{1}_{x>z}}
\end{aligned}
$$

Hence $f_{g}^{\prime}(x)=-2 \int_{-\infty}^{x} g(z) d z-2 k_{-} x f_{g}(x) \mathbf{1}_{x<0}-2 k_{+} x f_{g}(x) \mathbf{1}_{0 \leq x \leq m}$ and $f_{g}^{\prime}$ is continuous in 0 . Therefore $f_{g}$ is also continuous in 0 .

The second derivative of $f_{g}$ is $f_{g}^{\prime \prime}(x)=-2 g(x)-2 k_{-}\left(x f_{g}(x)\right)^{\prime} \mathbf{1}_{x<0}-2 k_{+}\left(x f_{g}(x)\right)^{\prime} \mathbf{1}_{0 \leq x \leq m}$, which concludes the proof.

To obtain that $T$ is well defined, continuous and compact, we first prove that the kernel $K$ is square-integrable. More precisely, one has

Lemma 2. The kernel $K$ satisfies $\left.\left.\left.\left.K \in L^{2}(]-\infty, m\right] \times\right]-\infty, m\right], e^{\frac{k(x) x^{2}-k(z) z^{2}}{2}}\right)$.

Proof. We have that

$$
\begin{aligned}
& \int_{-\infty}^{m} \int_{-\infty}^{m} e^{k(x) x^{2}-k(z) z^{2}} K^{2}(x, z) d x d z \\
& =\int_{-\infty}^{0} \int_{-\infty}^{0} e^{-k_{-} x^{2}-k_{-} z^{2}}\left(\int_{x \vee z}^{m} e^{k_{-} y^{2}} d y\right)^{2} d z d x+\underbrace{\int_{-\infty}^{0} e^{-k_{-} x^{2}} d x \int_{0}^{m} e^{-k_{+} z^{2}}\left(\int_{z}^{m} e^{k-y^{2}} d y\right)^{2} d z}_{<\infty} \\
& +\underbrace{\int_{0}^{m} \int_{0}^{m} e^{-k_{+} x^{2}-k_{+} z^{2}}\left(\int_{x \vee z}^{m} e^{k_{+} y^{2}} d y\right)^{2} d z d x}_{<\infty \text { on }[0, \mathrm{~m}]}+\underbrace{\int_{-\infty}^{0} e^{-k_{-} z^{2}} d z \int_{0}^{m} e^{-k_{+} x^{2}}\left(\int_{x}^{m} e^{k_{+} y^{2}} d y\right)^{2} d x}_{<\infty} .
\end{aligned}
$$

All we need to show now is that $I_{1}=\int_{-\infty}^{0} \int_{-\infty}^{0} e^{-k_{-} x^{2}-k_{-} z^{2}}\left(\int_{x \vee z}^{m} e^{k_{-} y^{2}} d y\right)^{2} d z d x$ is finite. Note that

$$
\begin{aligned}
I_{1} & =2 \int_{-\infty}^{0} e^{-k_{-} x^{2}}\left(\int_{-\infty}^{x} e^{-k_{-} z^{2}} d z\right)\left(\int_{x}^{m} e^{k_{-} y^{2}} d y\right)^{2} d x \\
& =C \int_{-\infty}^{0} e^{-k_{-} x^{2}} \Phi\left(\sqrt{2 k_{-}} x\right)\left(\int_{x}^{m} e^{k_{-} y^{2}} d y\right)^{2} d x,
\end{aligned}
$$

where $C$ is a constant. For some constants $C_{1}, C_{2}$, we have that Gaussian cdf $\Phi\left(\sqrt{2 k_{-}} x\right) \sim^{x \rightarrow-\infty} C_{1} \frac{e^{-k_{-} x^{2}}}{x}$ and $\left(\int_{x}^{m} e^{k_{-} y^{2}} d y\right)^{2} \sim^{x \rightarrow-\infty}\left(C_{2}-\frac{e^{k_{-} x^{2}}}{2 k_{-} x}\right)^{2}$, hence $I_{1}<$ $\infty$ and the conclusion holds.

Classical results on operators defined by a kernel imply 
Corollary 4. (a) The operator $\left.\left.\left.\left.\mathbf{T}: L^{2}(]-\infty, m\right], e^{k(.) .^{2} / 2}\right) \rightarrow L^{2}(]-\infty, m\right], e^{k(.) .^{2} / 2}\right)$ is well defined, continuous and compact.

(b) $\left.\left.L^{2}(]-\infty, m\right], e^{k(.)^{2} / 2}\right)$ admits an Hilbertian (countable) basis of eigenvectors of $\mathbf{T}$ for the scalar product associated to the $\left.\left.L^{2}(]-\infty, m\right], e^{k(.){ }^{2} / 2}\right)-n o r m$.

\section{Eigenvectors of $\mathbf{L}_{F P}$}

Let $\phi$ be an eigenvector of $\mathbf{L}_{F P}$ with eigenvalue $\mu$. Then, by the definition of $\mathbf{L}_{F P}$, we have for $x \leq m$

$$
\mathbf{L}_{F P} \phi(x)=\mu \phi(x) \Longleftrightarrow-(k(x) x \phi(x))^{\prime}-\frac{1}{2} \phi^{\prime \prime}(x)=\mu \phi(x) .
$$

If $\left.\left.\phi \in L^{2}(]-\infty, m\right], e^{k(.) .^{2} / 2}\right)$, then

$$
\left.\left.e^{\frac{k_{-} x^{2}}{2}} \phi(x) \mathbf{1}_{x \leq 0}+e^{\frac{k_{+} x^{2}}{2}} \phi(x) \mathbf{1}_{0<x \leq m}=\tilde{\phi}(x) \in L^{2}(]-\infty, m\right]\right) .
$$

Hence for $x \in \mathbb{R}_{-}$:

$$
\begin{gathered}
-\frac{1}{2}\left(e^{-\frac{k_{-} x^{2}}{2}} \tilde{\phi}(x)\right)^{\prime \prime}-k_{-}\left(x e^{-\frac{k_{-} x^{2}}{2}} \tilde{\phi}(x)\right)^{\prime}=\mu e^{-\frac{k_{-} x^{2}}{2}} \tilde{\phi}(x) \\
\Longleftrightarrow-\frac{1}{2} \tilde{\phi}^{\prime \prime}(x)+\frac{1}{2} k_{-}^{2} x^{2} \tilde{\phi}(x)=\left(\mu+\frac{k_{-}}{2}\right) \tilde{\phi}(x) .
\end{gathered}
$$

With the variable change $z=x \sqrt{2 k_{-}}$and $h(z)=\tilde{\phi}\left(\frac{z}{\sqrt{2 k_{-}}}\right)=\tilde{\phi}(x)$, we obtain the following equation satisfied by $h$ :

$$
h^{\prime \prime}(z)+\left(-\frac{1}{4} z^{2}+\frac{\mu}{k_{-}}+\frac{1}{2}\right) h(z)=0, z \leq 0 .
$$

Since $h$ is integrable on $-\infty$, then the solution is $h()=.D_{\frac{\mu}{k_{-}}}(-$.). We deduce that for $x \in \mathbb{R}_{-}, \phi(x)=e^{-\frac{k_{-}^{2} x^{2}}{2}} D_{\frac{\mu}{k_{-}}}\left(-x \sqrt{2 k_{-}}\right)$.

For $x \in] 0, m]$, it is easy to adapt the previous reasoning and there exist $\alpha, \beta \in \mathbb{R}$ such that

$$
\left.\left.\phi(x)=e^{-\frac{k_{+}^{2} x^{2}}{2}}\left[\alpha D_{\frac{\mu}{k_{+}}}\left(-x \sqrt{2 k_{+}}\right)+\beta D_{\frac{\mu}{k_{+}}}\left(x \sqrt{2 k_{+}}\right)\right] \quad \text { on }\right] 0, m\right] .
$$


Since $\phi \in D\left(\mathbf{L}_{F P}\right)$, it is a $C^{1}$-function in 0 . Therefore

$$
\begin{aligned}
& \alpha=\alpha_{\mu}=\frac{1}{2}\left[\frac{D_{\frac{\mu}{k_{-}}}(0)}{D_{\frac{\mu}{k_{+}}}(0)}+\sqrt{\frac{k_{+}}{k_{-}}} \frac{D_{\frac{\mu}{k_{-}}-1}(0)}{D_{\frac{\mu}{k_{+}}-1}(0)}\right] \\
& \beta=\beta_{\mu}=\frac{1}{2}\left[\frac{D_{\frac{\mu}{k_{-}}}(0)}{D_{\frac{\mu}{k_{+}}}(0)}-\sqrt{\frac{k_{+}}{k_{-}}} \frac{D_{\frac{\mu}{k_{-}}-1}(0)}{D_{\frac{\mu}{k_{+}}-1}(0)}\right] .
\end{aligned}
$$

Moreover $\phi(m)=0$, i.e. $\alpha_{\mu} D_{\frac{\mu}{k_{+}}}\left(-m \sqrt{2 k_{+}}\right)+\beta_{\mu} D_{\frac{\mu}{k_{+}}}\left(m \sqrt{2 k_{+}}\right)=0$.

In the following we denote by $\left(\mu_{n}\right)_{n \geq 1}$ the ordered sequence of zeros of $\mu \mapsto \alpha_{\mu} D_{\frac{\mu}{k_{+}}}\left(-m \sqrt{2 k_{+}}\right)+$ $\beta_{\mu} D_{\frac{\mu}{k_{+}}}\left(m \sqrt{2 k_{+}}\right)$.

Proposition 5. The roots $\left(\mu_{n}\right)_{n \geq 1}$ of $\alpha_{\mu} D_{\frac{\mu}{k_{+}}}\left(-m \sqrt{2 k_{+}}\right)+\beta_{\mu} D_{\frac{\mu}{k_{+}}}\left(m \sqrt{2 k_{+}}\right)=0$ are strictly positive.

Proof. Since $\mu_{n}$ is a zero of $\mu \mapsto \alpha_{\mu} D_{\frac{\mu}{k_{+}}}\left(-m \sqrt{2 k_{+}}\right)+\beta_{\mu} D_{\frac{\mu}{k_{+}}}\left(m \sqrt{2 k_{+}}\right)$, this is equivalent to a zero of $\mu \mapsto \alpha_{\mu} H_{\frac{\mu}{k_{+}}}\left(-m \sqrt{2 k_{+}}\right)+\beta_{\mu} H_{\frac{\mu}{k_{+}}}\left(m \sqrt{2 k_{+}}\right)$. So, using (13)

$$
\frac{1}{\Gamma\left(-\frac{\mu_{n}}{k_{+}}\right)} \sum_{i=0}^{\infty} \frac{1}{i !} \Gamma\left(\frac{i-\frac{\mu_{n}}{k_{+}}}{2}\right) \underbrace{\left[\left(2 m \sqrt{2 k_{+}}\right)^{i} \alpha_{n}+\left(-2 m \sqrt{2 k_{+}}\right)^{i} \beta_{n}\right]}_{:=A_{i}}=0 .
$$

Remark that

$$
\begin{aligned}
A_{2 i} & =\left(2 m \sqrt{2 k_{+}}\right)^{2 i}\left(\alpha_{n}+\beta_{n}\right)=\left(2 m \sqrt{2 k_{+}}\right)^{2 i} \times \frac{D_{\frac{\mu_{n}}{k_{-}}}(0)}{D_{\frac{\mu_{n}}{k_{+}}}(0)} \\
A_{2 i+1} & =\left(2 m \sqrt{2 k_{+}}\right)^{2 i+1}\left(\alpha_{n}-\beta_{n}\right)=\left(2 m \sqrt{2 k_{+}}\right)^{2 i+1} \times \sqrt{\frac{k_{+}}{k_{-}}} \frac{D_{\frac{\mu_{n}}{k_{-}}-1}(0)}{D_{\frac{\mu_{n}}{k_{+}}-1}(0)} .
\end{aligned}
$$

Using a proof by contradiction, let us assume that $\mu_{n}<0$ (hence $\frac{\mu_{n}}{k_{+}}, \frac{\mu_{n}}{k_{-}}, \frac{\mu_{n}}{k_{+}}-1, \frac{\mu_{n}}{k_{-}}-$ $1<0)$, then $\Gamma\left(-\frac{\mu_{n}}{k_{+}}\right)>0, \Gamma\left(\frac{i-\frac{\mu_{n}}{k_{+}}}{2}\right)>0, \frac{D \frac{\mu_{n}}{k_{-}}(0)}{D \frac{\mu_{n}}{k_{+}}(0)}>0$ and $\frac{D \frac{\mu_{n}}{k_{-}}-1}{D \frac{\mu_{n}}{k_{+}}(0)}>0$. Therefore the previous sum is strictly positive (all the terms are strictly positive). We deduce that $\mu_{n} \geq 0$.

Suppose now that $\mu_{n}=0$. Since $H_{0}(x)=1$, then $\mu_{n}$ will be solution of $\alpha_{n}+\beta_{n}=0$. This is absurde because when $\mu_{n}=0, \alpha_{n}+\beta_{n}=1$. We deduce that $\mu_{n}>0$.

\section{Expression of $p$}


We have found the eigenvectors of $\mathbf{L}_{F P}$ namely the functions

$x \mapsto e^{-\frac{k_{-}^{2} x^{2}}{2}} D_{\frac{\mu_{n}}{k_{-}}}\left(-x \sqrt{2 k_{-}}\right) \mathbf{1}_{x \leq 0}+e^{-\frac{k_{+}^{2} x^{2}}{2}}\left[\alpha_{n} D \frac{\mu_{n}}{k_{+}}\left(-x \sqrt{2 k_{+}}\right)+\beta_{n} D \frac{\mu_{n}}{k_{+}}\left(x \sqrt{2 k_{+}}\right)\right] \mathbf{1}_{0<x<m}$

with $\left(\mu_{n}\right)_{n \geq 1}$ being the ordered positive roots of $\mu \mapsto \alpha_{\mu} D_{\frac{\mu}{k_{+}}}\left(-m \sqrt{2 k_{+}}\right)+\beta_{\mu} D_{\frac{\mu}{k_{+}}}\left(m \sqrt{2 k_{+}}\right)$.

With a normalization, we finally have an Hilbertian basis $\left(e_{n}\right)_{n \geq 1}$ of $\left.\left.L^{2}(]-\infty, m\right], e^{k(.))^{2} / 2}\right)$ :

$e_{n}: x \mapsto \frac{e^{-\frac{k_{-}^{2} x^{2}}{2}} D_{\frac{\mu_{n}}{k_{-}}}\left(-x \sqrt{2 k_{-}}\right) \mathbf{1}_{x<0}+e^{-\frac{k_{+}^{2} x^{2}}{2}}\left[\alpha_{n} D_{\frac{\mu_{n}}{k_{+}}}\left(-x \sqrt{2 k_{+}}\right)+\beta_{n} D_{\frac{\mu_{n}}{k_{+}}}\left(x \sqrt{2 k_{+}}\right)\right] \mathbf{1}_{0<x<m}}{\int_{-\infty}^{0} D_{\frac{\mu_{n}}{k_{-}}}^{2}\left(-y \sqrt{2 k_{-}}\right) d y+\int_{0}^{m}\left[\alpha_{n} D_{\frac{\mu_{n}}{k_{+}}}\left(-y \sqrt{2 k_{+}}\right)+\beta_{n} D_{\frac{\mu_{n}}{k_{+}}}\left(y \sqrt{2 k_{+}}\right)\right]^{2} d y}$.

The following lemma gives another equivalent form of the denominator of $e_{n}$.

Lemma 3. Under the assumptions of Theorem 2, we have:

$$
\begin{gathered}
\int_{-\infty}^{0} D_{\frac{\mu_{n}}{k_{-}}}^{2}\left(-y \sqrt{2 k_{-}}\right) d y+\int_{0}^{m}\left[\alpha_{n} D_{\frac{\mu_{n}}{k_{+}}}\left(-y \sqrt{2 k_{+}}\right)+\beta_{n} D_{\frac{\mu_{n}}{k_{+}}}\left(y \sqrt{2 k_{+}}\right)\right]^{2} d y= \\
\frac{1}{2} \partial_{x}\left[\alpha_{n} D_{\frac{\mu_{n}}{k_{+}}}\left(-x \sqrt{2 k_{+}}\right)+\beta_{n} D_{\frac{\mu_{n}}{k_{+}}}\left(x \sqrt{2 k_{+}}\right)\right]_{x=m} \partial_{\mu}\left[\alpha_{\mu} D_{\frac{\mu}{k_{+}}}\left(-m \sqrt{2 k_{+}}\right)+\beta_{\mu} D_{\frac{\mu}{k_{+}}}\left(m \sqrt{2 k_{+}}\right)\right]_{\mu=\mu_{n}} .
\end{gathered}
$$

This term is denoted $N\left(\mu_{n}\right)$.

Proof. Using the same reasoning as [3] (see the proof of Proposition 6), we can prove that if $f: \mathbb{R}^{2} \rightarrow \mathbb{R}$ is solution of the following partial differential equation $\partial_{x^{2}}^{2} f(x, \mu)+\left(-x^{2} k^{2}+k+2 \mu\right) f(x, \mu)=0$, then for all $x, \mu, a, b \in \mathbb{R}$,

$$
\begin{gathered}
\int_{a}^{b} f^{2}(x, \mu) d x= \\
-\frac{1}{2}\left[f(b, \mu) \partial_{x} \partial_{\mu} f(b, \mu)-\partial_{x} f(b, \mu) \partial_{\mu} f(b, \mu)-f(a, \mu) \partial_{x} \partial_{\mu} f(a, \mu)+\partial_{x} f(a, \mu) \partial_{\mu} f(a, \mu)\right] .
\end{gathered}
$$

We apply this result for the two integrals. In the first case, we take $f(x, \mu)=$ $D_{\frac{\mu}{k_{-}}}\left(-x \sqrt{2 k_{+}}\right)$and for the second integral $f(x, \mu)=\alpha_{\mu} D_{\frac{\mu}{k_{+}}}\left(-x \sqrt{2 k_{+}}\right)+\beta_{\mu} D_{\frac{\mu}{k_{+}}}\left(x \sqrt{2 k_{+}}\right)$.

We conclude using the relations between $\alpha_{n}$ and $\beta_{n}$.

We look for a solution $p$ of (5) that belongs to $\left.\left.L^{2}(]-\infty, m\right], e^{k(.))^{2} / 2}\right)$. We can decompose $p$ on the basis $\left(e_{n}\right)_{n \geq 1}$ :

$$
p(m, x, t)=\sum_{n=1}^{\infty} c_{n}(t) e_{n}(x) .
$$


However $p(m, x, 0)=\sum_{n=1}^{\infty} c_{n}(0) e_{n}(x)$, then for $n \in \mathbb{N}^{*}$ :

$$
\begin{aligned}
c_{n}(0) & =<p(m, ., 0), e_{n}>_{\left.\left.L^{2}(]-\infty, m\right], e^{k(.) .^{2} / 2}\right)} \\
& =\int_{-\infty}^{0} e^{k_{-} z^{2}} p(m, z, 0) e_{n}(z) d z+\int_{0}^{m} e^{k_{+} z^{2}} p(m, z, 0) e_{n}(z) d z \\
& =\frac{e^{\frac{k_{-} x_{0}^{2}}{2}} D_{\frac{\mu_{n}}{k_{-}}}\left(-x_{0} \sqrt{2 k_{-}}\right) \mathbf{1}_{x_{0} \leq 0}+e^{\frac{k_{+} x_{0}^{2}}{2}}\left[\alpha_{n} D \frac{\mu_{n}}{k_{+}}\left(-x_{0} \sqrt{2 k_{+}}\right)+\beta_{n} D_{\frac{\mu_{n}}{k_{+}}}\left(x_{0} \sqrt{2 k_{+}}\right)\right] \mathbf{1}_{0<x \leq m}}{N\left(\mu_{n}\right)}
\end{aligned}
$$

It remains to find $c_{n}(t)$ for $t>0$. On the one hand

$$
\partial_{t} p(m, x, t)=\sum_{n=1}^{\infty} c_{n}^{\prime}(t) e_{n}(x)
$$

and on the other hand

$$
\partial_{t} p(m, x, t)=-\mathbf{L}_{F P} p(m, x, t)=-\sum_{n=1}^{\infty} c_{n}(t) \mathbf{L}_{F P} e_{n}(x)=-\sum_{n=1}^{\infty} c_{n}(t) \mu_{n} e_{n}(x) .
$$

The decomposition is unique, thus $c_{n}^{\prime}(t)=-c_{n}(t) \mu_{n}$ and $c_{n}(t)=c_{n}(0) e^{-\mu_{n} t}$.

\subsection{Proof of Theorem 1}

First of all recall that if $\left(Y_{t}\right)_{t \geq 0}$ is a Brownian Motion $(\mathrm{BM}), y \in \mathbb{R}, \tilde{P}_{y}$ the law of $\left(Y_{t}\right)_{t \geq 0}$ when $Y_{0}=y$ and $\left(\mathcal{F}_{t}\right)_{t \geq 0}$ the filtration generated by the BM, then Girsanov Theorem gives:

$$
\forall t>0,\left.\quad \frac{\mathrm{d} \mathbb{P}_{y}}{\mathrm{~d} \tilde{\mathbb{P}}_{y}}\right|_{\mathcal{F}_{t}}=\exp \left(-\int_{0}^{t} k\left(Y_{s}\right) Y_{s} \mathrm{~d} Y_{s}-\frac{1}{2} \int_{0}^{t}\left(k\left(Y_{s}\right)\right)^{2} Y_{s}^{2} \mathrm{~d} s\right),
$$

and $W_{t}:=Y_{t}+\int_{0}^{t} k\left(Y_{s}\right) Y_{s} \mathrm{~d} s$ is a BM under $\mathbb{P}_{y}$ and $\left(Y_{t}\right)_{t \geq 0}$ is an OU process with broken drift

$$
\mathrm{d} Y_{t}=-k\left(Y_{t}\right) Y_{t} \mathrm{~d} t+\mathrm{d} W_{t}
$$

1. The proof of the existence of $p_{\tau}$ is similar to [17]. Let $h: \mathbb{R} \rightarrow \mathbb{R}$ a function defined by $h(x)=-\int_{0}^{x} k(s) s d s$. The derivative of $h$ is absolutely continuous. Ito formula gives (we can apply it, see Problem 7.3 , Section 3.7 [10])

$$
h\left(X_{t}\right)=h\left(X_{0}\right)-\int_{0}^{t} k\left(X_{s}\right) X_{s} d X_{s}+\frac{1}{2} \int_{0}^{t} h^{\prime \prime}\left(X_{s}\right) d s, t \in \mathbb{R}_{+}
$$

where $h^{\prime \prime}=-\left(k_{-} \mathbf{1}_{\mathbb{R}_{-}^{*}}+k_{+} \mathbf{1}_{\mathbb{R}_{+}^{*}}\right)$ exists a.e. 
Let $T>0$ and $f$ a function $\mathcal{C}\left(\mathbb{R}_{+}, \mathbb{R}\right)$ such that $\operatorname{supp}(f) \subset[0, T]$ :

$$
\begin{aligned}
\mathbb{E}_{x}[f(\tau)] & =\tilde{\mathbb{E}}_{x}\left[f(\tau \wedge T) \exp \left(-\int_{0}^{T} k\left(X_{s}\right) X_{s} \mathrm{~d} X_{s}-\frac{1}{2} \int_{0}^{T}\left(k\left(X_{s}\right)\right)^{2} X_{s}^{2} \mathrm{~d} s\right)\right] \\
& =\tilde{\mathbb{E}}_{x}\left[f(\tau) \exp \left(h\left(X_{T}\right)-h\left(X_{0}\right)-\frac{1}{2} \int_{0}^{T} h^{\prime \prime}\left(X_{s}\right)+\left(k\left(X_{s}\right)\right)^{2} X_{s}^{2} \mathrm{~d} s\right)\right] \\
& =\int_{0}^{T} f(s) \int_{-\infty}^{+\infty} e^{h(\xi)-h(x)} \tilde{\mathbb{E}}_{x}\left[e^{-\frac{1}{2} \int_{0}^{T} g\left(X_{u}\right) d u} \mid \tau=s, X_{T}=\xi\right] \tilde{\mathbb{P}}_{x}\left(\tau \in \mathrm{d} s, X_{T} \in \mathrm{d} \xi\right) .
\end{aligned}
$$

where $g(x)=h^{\prime \prime}(x)+k(x)^{2} x^{2}, x \in \mathbb{R}^{*}$. Since $\left(X_{t}\right)_{t \geq 0}$ is a BM under $\tilde{\mathbb{P}}_{x}$ :

$$
\begin{aligned}
\tilde{\mathbb{P}}_{x}\left(\tau \in \mathrm{d} s, X_{T} \in \mathrm{d} \xi\right) & =\tilde{\mathbb{P}}_{x}(\tau \in \mathrm{d} s) \tilde{\mathbb{P}}_{x}\left(X_{T-s}+m \in \mathrm{d} \xi\right) \\
& =\frac{|m-x|}{\sqrt{2 \pi s^{3}}} e^{-\frac{(m-x)^{2}}{2 s}} \mathrm{~d} s \frac{1}{\sqrt{2 \pi(T-s)}} e^{-\frac{(\xi-m-x)^{2}}{2(T-s)}} \mathrm{d} \xi
\end{aligned}
$$

and we easily conclude that the density of $\tau$ exists.

2. We use the link between $p_{\tau}$ and $p$, namely: $p_{\tau}(t)=-\left.\frac{1}{2} \partial_{x} p(m, x, t)\right|_{x=m}$. Indeed,

$$
\begin{aligned}
p_{\tau}(t) & =-\partial_{t} \mathbb{P}(\tau>t)=-\partial_{t} \int_{-\infty}^{m} p(m, x, t) d x \\
& =-\int_{-\infty}^{m} \partial_{x}(k(x) x p(m, x, t))+\frac{1}{2} \partial_{x}^{2} p(m, x, t) d x \\
& =-\left[k(x) x p(m, x, t)+\frac{1}{2} \partial_{x} p(m, x, t)\right]_{-\infty}^{m}=-\left.\frac{1}{2} \partial_{x} p(m, x, t)\right|_{x=m}
\end{aligned}
$$

We consider here only the case $x_{0} \in \mathbb{R}_{-}$(for $\left.\left.x_{0} \in\right] 0, m\right]$ we use similar arguments). We use Formula (6), case 2, for computing the derivative $\frac{\partial}{\partial x} p(m, x, t)$ at $x=m$. Since we are given an infinite sum, we have to prove that

$$
\sum_{n=1}^{\infty} \frac{e^{-\mu_{n} t}}{N\left(\mu_{n}\right)} D \frac{\mu_{n}}{k_{-}}\left(-x_{0} \sqrt{2 k_{-}}\right) \partial_{x}\left[\alpha_{n} D \frac{\mu_{n}}{k_{+}}\left(-x \sqrt{2 k_{+}}\right)+\beta_{n} D \frac{\mu_{n}}{k_{+}}\left(x \sqrt{2 k_{+}}\right)\right]
$$

converges uniformly in a neighborhood of $\mathrm{m}$. Consider a neighborhood of the form $[m-\varepsilon, m]$ with $m-\varepsilon \geq 0$. Using the expression (8) of $N\left(\mu_{n}\right)$ and Corollary 6 in the appendix, we obtain for all $x \in[m-\varepsilon, m]$

$$
\left|\frac{\partial_{x}\left[\alpha_{n} D \frac{\mu_{n}}{k_{+}}\left(-x \sqrt{2 k_{+}}\right)+\beta_{n} D \frac{\mu_{n}}{k_{+}}\left(x \sqrt{2 k_{+}}\right)\right]}{N\left(\mu_{n}\right)}\right| \leq\left|\frac{1}{\partial_{\mu}\left[\alpha_{\mu} D \frac{\mu}{k_{+}}\left(-m \sqrt{2 k_{+}}\right)+\beta_{\mu} D_{\frac{\mu}{k_{+}}}\left(m \sqrt{2 k_{+}}\right)\right]_{\mu=\mu_{n}}}\right| .
$$


Thus, for proving that the sum (10) is uniformly convergent it is sufficient to show that

$$
\sum_{n=1}^{\infty}\left|e^{-\mu_{n} t} \frac{D_{\frac{\mu_{n}}{k_{-}}}\left(-x_{0} \sqrt{2 k_{-}}\right)}{\partial_{\mu}\left[\alpha_{\mu} D_{\frac{\mu}{k_{+}}}\left(-m \sqrt{2 k_{+}}\right)+\beta_{\mu} D_{\frac{\mu}{k_{+}}}\left(m \sqrt{2 k_{+}}\right)\right]_{\mu=\mu_{n}}}\right|<\infty .
$$

To complete the proof it suffices to apply (21) and (22) below. Thus for large $n$ we have

$$
\left|e^{-\mu_{n} t} \frac{D_{\frac{\mu_{n}}{k_{-}}}\left(-x_{0} \sqrt{2 k_{-}}\right)}{\partial_{\mu}\left[\alpha_{\mu} D_{\frac{\mu}{k_{+}}}\left(-m \sqrt{2 k_{+}}\right)+\beta_{\mu} D_{\frac{\mu}{k_{+}}}\left(m \sqrt{2 k_{+}}\right)\right]_{\mu=\mu_{n}}}\right| \stackrel{n \rightarrow \infty}{\leq} C e^{-k_{-}(2 n-1) t},
$$

with $C$ constant.

\subsection{Proof of Corollary 3}

Notice that

$$
P\left(\sup _{0 \leq u \leq t} X_{u}<m\right)=P(\tau>t)=\int_{t}^{\infty} p_{\tau}(s) d s,
$$

and hence we obtain the formula of Corollary 3 by integrating the density (4) over $[t, \infty)$. Observe that we can interchange the integral and the infinite sum, because the series on RHS of Equation (4) is absolutely convergent. For the case $x_{0} \in \mathbb{R}_{-}$this follows from (12) and the fact that the sequence $\left(\mu_{n}\right)_{n}$ converges to infinity (see (21) below).

\section{Appendix}

\subsection{Definitions and some properties on Hermite functions and parabolic cylinder functions}

We recall here the definitions and some known results about parabolic cylinder functions and Hermite functions. These results can be found in Chapters 4 and 10 of [11] and in Section 7 of [2].

For $\nu \in \mathbb{R}$, the Hermite function $H_{\nu}$ is defined, for all $x \in \mathbb{R}$, by

$$
H_{\nu}(x)=\frac{1}{2 \Gamma(-\nu)} \sum_{i=0}^{+\infty} \frac{(-1)^{i}}{i !} \Gamma\left(\frac{i-\nu}{2}\right)(2 x)^{i},
$$

or, equivalently, by

$$
H_{\nu}(x)=2^{\nu}\left[\frac{\Gamma\left(\frac{1}{2}\right)}{\Gamma\left(\frac{1-\nu}{2}\right)}{ }_{1} F_{1}\left(\frac{-\nu}{2}, \frac{1}{2}, x^{2}\right)+x \frac{\Gamma\left(-\frac{1}{2}\right)}{\Gamma\left(\frac{-\nu}{2}\right)}{ }_{1} F_{1}\left(\frac{1-\nu}{2}, \frac{3}{2}, x^{2}\right)\right],
$$


where ${ }_{1} F_{1}$ is the Kummer confluent hypergeometric function. One can show that

$$
H_{\nu}(0)=\frac{2^{\nu} \Gamma\left(\frac{1}{2}\right)}{\Gamma\left(\frac{1-\nu}{2}\right)}, \quad H_{\nu}^{\prime}(0)=\frac{2^{\nu} \Gamma\left(-\frac{1}{2}\right)}{\Gamma\left(\frac{-\nu}{2}\right)} .
$$

The Hermite function satisfies the following relations:

$$
\partial_{x} H_{\nu}(x)=2 \nu H_{\nu-1}(x)
$$

and

$$
\partial_{x}^{2} H_{\nu}(x)=-2 \nu H_{\nu}(x)+2 x \partial_{x} H_{\nu}(x) .
$$

For $\nu \in \mathbb{R}$ we define the parabolic cylinder function as the function

$$
D_{\nu}(x)=2^{-\frac{\nu}{2}} e^{-\frac{x^{2}}{4}} H_{\nu}\left(\frac{x}{\sqrt{2}}\right), \quad x \in \mathbb{R} .
$$

One can show that $D_{\nu}$ is one of the two solutions of the differential equation

$$
y^{\prime \prime}(x)+\left(-\frac{x^{2}}{4}+\frac{1}{2}+\nu\right) y(x)=0, \quad x \in \mathbb{R} .
$$

Notice that the second solution of (19) is given by $x \mapsto D_{\nu}(-x)$. We have the following relation:

$$
\partial_{x} D_{\nu}(x)=\nu D_{\nu-1}(x)-\frac{x}{2} D_{\nu}(x) .
$$

Finally, we have the following asymptotic expansion:

$$
H_{\nu}(x)=\frac{2^{\nu} \Gamma\left(\frac{1+\nu}{2}\right) e^{\frac{x^{2}}{2}}}{\sqrt{\pi}}\left[\cos \left(x \sqrt{2 \nu+1}-\frac{\pi \nu}{2}\right)+O\left(\nu^{-\frac{1}{4}}\right)\right] .
$$

\subsection{Asymptotic expansions for large $\mu$}

For the proof of Theorem 1 we need some asymptotic properties of the function defined by

$$
G(x, \mu)=\alpha_{\mu} D_{\frac{\mu}{k_{+}}}\left(-x \sqrt{2 k_{+}}\right)+\beta_{\mu} D_{\frac{\mu}{k_{+}}}\left(x \sqrt{2 k_{+}}\right) .
$$

We start by deriving the asymptotic behaviour of $G(x, \mu)$ as $\mu \rightarrow \infty$ (cf. the method given in [18] p. 69 for the function $\phi$ ).

Lemma 4. When $\mu \rightarrow \infty$, the following asymptotic formula holds:

$$
G(x, \mu)=2^{\frac{\mu}{2 k_{-}}} \frac{\Gamma\left(\frac{1}{2}+\frac{\mu}{2 k_{-}}\right)}{\sqrt{\pi}}\left[\cos \left(x \sqrt{k_{+}+2 \mu}-\frac{\pi \mu}{2 k_{-}}\right)+O\left(\mu^{-\frac{1}{4}}\right)\right] .
$$


Sketch of the proof. Using (14), (18), and the definition of $\alpha_{\mu}$ and $\beta_{\mu}$, we find:

$G(x, \mu)=2^{\frac{\mu}{2 k_{-}}} e^{-\frac{x^{2} k_{+}}{2}}\left[\frac{\sqrt{\pi}}{\Gamma\left(\frac{1-\frac{\mu}{k_{-}}}{2}\right)}{ }_{1} F_{1}\left(-\frac{\mu}{2 k_{+}}, \frac{1}{2}, x^{2} k_{+}\right)+\frac{2 \sqrt{\pi} x \sqrt{k_{-}}}{\Gamma\left(-\frac{\mu}{2 k_{-}}\right)}{ }_{1} F_{1}\left(\frac{1-\frac{\mu}{k_{+}}}{2}, \frac{3}{2}, x^{2} k_{+}\right)\right]$.

Tricomi's formula (see for ex. [18], p.57) gives

$$
\begin{aligned}
G(x, \mu)= & 2^{\frac{\mu}{2 k_{-}}}\left(x^{2} k_{+}\left(\frac{1}{4}+\frac{\mu}{2 k_{+}}\right)\right)^{\frac{1}{4}} \Gamma\left(\frac{1}{2}+\frac{\mu}{2 k_{-}}\right) \sum_{n=0}^{\infty}\left(\frac{x^{2} k_{+}}{\frac{2 \mu}{k_{+}}+1}\right)^{\frac{n}{2}} \times \\
& \times\left[A_{n}\left(\frac{1}{4}+\frac{\mu}{2 k_{+}}, \frac{1}{4}\right) \cos \left(\frac{\mu \pi}{2 k_{-}}\right) J_{-\frac{1}{2}+n}\left(x \sqrt{2 \mu+k_{+}}\right)\right. \\
& \left.-A_{n}\left(\frac{1}{4}+\frac{\mu}{2 k_{+}}, \frac{3}{4}\right) \sin \left(\frac{\mu \pi}{2 k_{-}}\right) J_{\frac{1}{2}+n}\left(x \sqrt{2 \mu+k_{+}}\right) \frac{\sqrt{k_{-}} \Gamma\left(1+\frac{\mu}{2 k_{-}}\right)\left(\frac{1}{4}+\frac{\mu}{2 k_{+}}\right)^{-\frac{1}{2}}}{\sqrt{k_{+}} \Gamma\left(\frac{1}{2}+\frac{\mu}{2 k_{-}}\right)}\right],
\end{aligned}
$$

where $J$ is the Bessel function and the coefficients $A_{n}$ are recursively defined by

$$
\begin{gathered}
(n+1) A_{n+1}\left(k, \frac{b}{2}\right)=(n+b-1) A_{n-1}\left(k, \frac{b}{2}\right)-2 k A_{n-2}\left(k, \frac{b}{2}\right), \\
A_{0}=1, A_{1}=0, A_{2}=\frac{b}{2} .
\end{gathered}
$$

Hence, we can write

$$
G(x, \mu)=2^{\frac{\mu}{2 k_{-}}}\left(x^{2} k_{+}\left(\frac{1}{4}+\frac{\mu}{2 k_{+}}\right)\right)^{\frac{1}{4}} \Gamma\left(\frac{1}{2}+\frac{\mu}{2 k_{-}}\right) \sum_{n=0}^{\infty} U_{n},
$$

where

$$
U_{n}=u_{3 n}+u_{3 n+1}+u_{3 n+2}
$$

and

$$
\begin{aligned}
u_{n}= & \left(\frac{x^{2} k_{+}}{\frac{2 \mu}{k_{+}}+1}\right)^{\frac{n}{2}}\left[A_{n}\left(\frac{1}{4}+\frac{\mu}{2 k_{+}}, \frac{1}{4}\right) \cos \left(\frac{\mu \pi}{2 k_{-}}\right) J_{-\frac{1}{2}+n}\left(x \sqrt{2 \mu+k_{+}}\right)\right. \\
& \left.-A_{n}\left(\frac{1}{4}+\frac{\mu}{2 k_{+}}, \frac{3}{4}\right) \sin \left(\frac{\mu \pi}{2 k_{-}}\right) J_{\frac{1}{2}+n}\left(x \sqrt{2 \mu+k_{+}}\right) \frac{\sqrt{k_{-}} \Gamma\left(1+\frac{\mu}{2 k_{-}}\right)\left(\frac{1}{4}+\frac{\mu}{2 k_{+}}\right)^{-\frac{1}{2}}}{\sqrt{k_{+}} \Gamma\left(\frac{1}{2}+\frac{\mu}{2 k_{-}}\right)}\right] .
\end{aligned}
$$


Since $\left|\cos \left(\frac{\mu \pi}{2 k_{-}}\right) J_{-\frac{1}{2}+n}\left(x \sqrt{2 \mu+k_{+}}\right)\right| \leq 1,\left|\sin \left(\frac{\mu \pi}{2 k_{-}}\right) J_{\frac{1}{2}+n}\left(x \sqrt{2 \mu+k_{+}}\right)\right| \leq 1$ and $\frac{\sqrt{k_{-}} \Gamma\left(1+\frac{\mu}{2 k_{-}}\right)\left(\frac{1}{4}+\frac{\mu}{2 k_{+}}\right)^{-\frac{1}{2}}}{\sqrt{k_{+}} \Gamma\left(\frac{1}{2}+\frac{\mu}{2 k_{-}}\right)}=1+O\left(\mu^{-1}\right)$, then

$$
\begin{aligned}
u_{3 n} & =O\left(\mu^{-\frac{n}{2}}\right) \\
u_{3 n+1} & =O\left(\mu^{-\frac{n}{2}-\frac{3}{2}}\right) \\
u_{3 n+2} & =O\left(\mu^{-\frac{n}{2}-1}\right)
\end{aligned}
$$

Hence $U_{n}=O\left(\mu^{-\frac{n}{2}}\right)$. Thus $U_{n} \rightarrow 0$ as $\mu \rightarrow \infty$. Moreover we can prove that $U_{n}=$ $O\left(\mu^{-\frac{n}{2}}\right) \leq C(x) \mu^{-\frac{n}{2}}$ for all $n$. Then we can deduce that $\sum_{n=0}^{\infty} U_{n}=U_{0}+O\left(\mu^{-\frac{1}{2}}\right)$. Hence

$$
G(x, \mu)=2^{\frac{\mu}{2 k_{-}}}\left(x^{2} k_{+}\left(\frac{1}{4}+\frac{\mu}{2 k_{+}}\right)\right)^{\frac{1}{4}} \Gamma\left(\frac{1}{2}+\frac{\mu}{2 k_{-}}\right)\left(U_{0}+O\left(\mu^{-\frac{1}{2}}\right)\right) .
$$

From the equalities $J_{-\frac{1}{2}}\left(x \sqrt{2 \mu+k_{+}}\right)=\left(\frac{2}{\pi x \sqrt{2 \mu+k_{+}}}\right)^{\frac{1}{2}} \cos \left(x \sqrt{2 \mu+k_{+}}\right)$and $J_{\frac{1}{2}}\left(x \sqrt{2 \mu+k_{+}}\right)=$ $\left(\frac{2}{\pi x \sqrt{2 \mu+k_{+}}}\right)^{\frac{1}{2}} \sin \left(x \sqrt{2 \mu+k_{+}}\right)$, one can get
$U_{0}=\left(\frac{2}{\pi x \sqrt{2 \mu+k_{+}}}\right)^{\frac{1}{2}} \cos \left(x \sqrt{k_{+}+2 \mu}-\frac{\pi \mu}{2 k_{-}}\right)+O\left(\mu^{-\frac{5}{4}}\right)$.

We finally find

$$
G(x, \mu)=2^{\frac{\mu}{2 k_{-}}} \frac{\Gamma\left(\frac{1}{2}+\frac{\mu}{2 k_{-}}\right)}{\sqrt{\pi}}\left[\cos \left(x \sqrt{k_{+}+2 \mu}-\frac{\pi \mu}{2 k_{-}}\right)+O\left(\mu^{-\frac{1}{4}}\right)\right] .
$$

For large $\mu$, the zeros of $G(x, \mu)$ are the zeros of $U_{0}$ which are very close of the zeros of $\cos \left(x \sqrt{k_{+}+2 \mu}-\frac{\pi \mu}{2 k_{-}}\right)$. Hence, for large $n, \mu_{n}$ is close of the positive solution $m \sqrt{k_{+}+2 \mu}-\frac{\pi \mu}{2 k_{-}}=\left(-n+\frac{1}{2}\right) \pi$ and we have the asymptotics :

$$
\begin{aligned}
& \mu_{n,+} \sim^{n \rightarrow \infty} k_{-}(2 n-1)+\frac{4 m^{2} k_{-}^{2}}{\pi^{2}}+\frac{2 m k_{-}}{\pi} \sqrt{\frac{4 m^{2} k_{-}^{2}}{\pi^{2}}+2 k_{-}(2 n-1)+k_{+}} \\
& \mu_{n,-} \sim^{n \rightarrow \infty} k_{-}(2 n-1)+\frac{4 m^{2} k_{-}^{2}}{\pi^{2}}-\frac{2 m k_{-}}{\pi} \sqrt{\frac{4 m^{2} k_{-}^{2}}{\pi^{2}}+2 k_{-}(2 n-1)+k_{+}}
\end{aligned}
$$

For $\mu$ in a neighborhood of a zero $\mu_{n}$, we can prove that

$$
G(x, \mu)=2^{\frac{\mu}{2 k_{-}}} \frac{\Gamma\left(\frac{1}{2}+\frac{\mu}{2 k_{-}}\right)}{\sqrt{\pi}} F(x, \mu)\left[1+O\left(\mu^{-\frac{1}{4}}\right)\right],
$$


where $F(x, \mu)=\cos \left(\frac{\mu \pi}{2 k_{-}}\right) \cos \left(x \sqrt{k_{+}+2 \mu}\right)+\sin \left(\frac{\mu \pi}{2 k_{-}}\right) \sin \left(x \sqrt{k_{+}+2 \mu}\right) \frac{\sqrt{k_{-}} \Gamma\left(1+\frac{\mu}{2 k_{-}}\right)\left(\frac{1}{4}+\frac{\mu}{2 k_{+}}\right)^{-\frac{1}{2}}}{\sqrt{k_{+}} \Gamma\left(\frac{1}{2}+\frac{\mu}{2 k_{-}}\right)}$.

Since for large $n, \mu_{n}$ is solution of $F\left(m, \mu_{n}\right)=0$, we have

$$
\begin{aligned}
\partial_{\mu} G\left(m, \mu_{n}\right)= & \partial_{\mu}\left(2^{\frac{\mu}{2 k_{-}}} \frac{\Gamma\left(\frac{1}{2}+\frac{\mu}{2 k_{-}}\right)}{\sqrt{\pi}}\left(1+O\left(\mu^{-\frac{1}{4}}\right)\right)\right) \underbrace{F\left(m, \mu_{n}\right)}_{\mu=\mu_{n}} \\
& +2^{\frac{\mu_{n}}{2 k_{-}}} \frac{\Gamma\left(\frac{1}{2}+\frac{\mu_{n}}{2 k_{-}}\right)}{\sqrt{\pi}}\left(1+O\left(\mu_{n}^{-\frac{1}{4}}\right)\right) \underbrace{\partial_{\mu} F(m, \mu)_{\mu=\mu_{n}}}_{=(-1)^{n} \frac{\pi}{2 k_{-}}+O\left(\mu_{n}^{-\frac{1}{2}}\right)} \\
= & 2^{\frac{\mu_{n}}{2 k_{-}}} \frac{\sqrt{\pi} \Gamma\left(\frac{1}{2}+\frac{\mu_{n}}{2 k_{-}}\right)}{2 k_{-}}(-1)^{n}\left(1+O\left(\mu_{n}^{-\frac{1}{4}}\right)\right) .
\end{aligned}
$$

Hence :

$$
\frac{D_{\frac{\mu_{n}}{k_{-}}}\left(-x_{0} \sqrt{2 k_{-}}\right)}{\partial_{\mu} G\left(m, \mu_{n}\right)}=\frac{(-1)^{n} e^{-x_{0}^{2} k_{-}} 2 k_{-}}{\pi} \cos \left(x_{0} \sqrt{k_{-}+2 \mu_{n}}+\frac{\pi \mu_{n}}{2 k_{-}}\right)+O\left(\mu_{n}^{-\frac{1}{4}}\right) .
$$

\subsection{Auxiliary results}

Lemma 5. Let $\nu, m, \varepsilon \in \mathbb{R}_{+}$such that $m-\varepsilon \geq 0$. For all $\alpha, \beta \in \mathbb{R}$ and $x \in[m-\varepsilon, m]$, we have:

$$
\left|\alpha H_{\nu-1}(-x)+\beta H_{\nu-1}(x)\right| \leq \sqrt{f(m)}
$$

where $f(x)=\left[\alpha H_{\nu-1}(-x)+\beta H_{\nu-1}(x)\right]^{2}+\frac{1}{2 \nu}\left[-\alpha H_{\nu}(-x)+\beta H_{\nu}(x)\right]^{2}$.

Proof. We apply the method given in [1]. Since

$$
\left(-\alpha H_{\nu}(-x)+\beta H_{\nu}(x)\right)^{\prime}=2 \nu\left(\alpha H_{\nu-1}(-x)+\beta H_{\nu-1}(x)\right),
$$

we have $f(x)=\frac{1}{4 \nu^{2}}\left\{\left[\left(-\alpha H_{\nu}(-x)+\beta H_{\nu}(x)\right)^{\prime}\right]^{2}+2 \nu\left[-\alpha H_{\nu}(-x)+\beta H_{\nu}(x)\right]^{2}\right\}$.

Using the fact that $-\alpha H_{\nu}(-x)+\beta H_{\nu}(x)$ is a solution of $y^{\prime \prime}(x)+2 \nu y(x)-2 x y^{\prime}(x)=0$, we see that

$$
f^{\prime}(x)=\frac{x\left[\left(-\alpha H_{\nu}(-x)+\beta H_{\nu}(x)\right)^{\prime}\right]^{2}}{\nu^{2}} .
$$

Hence $f$ is a decreasing function on $\mathbb{R}_{-}$and increasing on $\mathbb{R}_{+}$. We deduce that for all $x \in[m-\varepsilon, m]$ and $m-\varepsilon \geq 0$,

$$
\left[\alpha H_{\nu-1}(-x)+\beta H_{\nu-1}(x)\right]^{2} \leq f(x) \leq f(m),
$$

and the conclusion hold.

A direct consequence of this lemma is the following result : 
Corollary 6. Let $m, \varepsilon \geq 0$ such that $m-\varepsilon \geq 0$. If $\nu \geq 0$ is a zero of $\nu \mapsto \alpha H_{\nu}(-m)+$ $\beta H_{\nu}(m)$, then for all $x \in[m-\varepsilon, m]$ We have :

$$
\left|\left(\alpha H_{\nu}(-x)+\beta H_{\nu}(x)\right)^{\prime}\right| \leq\left|\left(\alpha H_{\nu}(-x)+\beta H_{\nu}(x)\right)^{\prime}\right|_{x=m} .
$$

\section{References}

[1] A. Alexandrov and G. Nikolov. An inequality of Duffin-Schaeffer type for Hermite polynomials. In Constructive theory of functions, pages 9-20. Prof. M. Drinov Acad. Publ. House, Sofia, 2012.

[2] L. Alili, P. Patie, and J. L. Pedersen. Representations of the first hitting time density of an Ornstein-Uhlenbeck process. Stoch. Models, 21(4):967-980, 2005.

[3] C. Blanchet-Scalliet, D. Dorobantu, and L. Gay. Joint law of an Ornstein-Uhlenbeck process and its supremum. J. Appl. Probab., 57(2):541-558, 2020.

[4] S. Browne and W. Whitt. Piecewise-linear diffusion processes. In Advances in queueing, Probab. Stochastics Ser., pages 463-480. CRC, Boca Raton, FL, 1995.

[5] F. Delarue, J. Inglis, S. Rubenthaler, and E. Tanré. First hitting times for general non-homogeneous 1d diffusion processes: density estimates in small time. hal00870991, 2013.

[6] P. V. Gapeev and N. Rodosthenous. Perpetual American options in a diffusion model with piecewise-linear coefficients. Stat. Risk Model., 30(1):1-20, 2013.

[7] L. Gay. Processus d'Ornstein-Uhlenbeck et son supremum: quelques résultats théoriques et application au risque climatique. PhD thesis, Université de Lyon, 2019.

[8] D. Guterding and W. Boenkost. The Heston stochastic volatility model with piecewise constant parameters - efficient calibration and pricing of window barrier options. J. Comput. Appl. Math., 343:353-362, 2018.

[9] M. Jeanblanc, M. Yor, and M. Chesney. Mathematical methods for financial markets. Springer Finance. Springer-Verlag London, Ltd., London, 2009.

[10] I. Karatzas and S. E. Shreve. Brownian motion and stochastic calculus, volume 113 of Graduate Texts in Mathematics. Springer-Verlag, New York, second edition, 1991.

[11] N. Lebedev. Special functions and their applications. Dover Publications, Inc., New York, 1972. Revised edition, translated from the Russian and edited by Richard A. Silverman, Unabridged and corrected republication.

[12] A. Lejay and P. Pigato. Maximum likelihood drift estimation for a threshold diffusion. Scandinavian Journal of Statistics, 47(3):609-637, 2020. 
[13] V. Linetsky. Computing hitting time densities for CIR and OU diffusions: Applications to mean-reverting models. Journal of Computational Finance, 7:1-22, 2004.

[14] J. M. McNamara. Optimal control of the diffusion coefficient of a simple diffusion process. Math. Oper. Res., 8(3):373-380, 1983.

[15] E. Mordecki and P. Salminen. Optimal stopping of brownian motion with broken drift. High Frequency, 2(2):113-120, 2019.

[16] E. Mordecki and P. Salminen. Optimal stopping of oscillating Brownian motion. Electron. Commun. Probab., 24:Paper No. 50, 12, 2019.

[17] E. J. Pauwels. Smooth first-passage densities for one-dimensional diffusions. J. Appl. Probab., 24(2):370-377, 1987.

[18] L. J. Slater. Confluent hypergeometric functions. Cambridge University Press, New York, 1960. 